\title{
Oleanolic acid inhibits osteosarcoma cell proliferation and invasion by suppressing the SOX9/Wnt1 signaling pathway
}

\author{
XIANMING CHEN $^{1 *}$, YULIN ZHANG $^{2 *}$, SEN ZHANG $^{1}$, AIMING WANG $^{1}$, QUANYIN DU $^{1}$ and ZIMING WANG ${ }^{1}$ \\ ${ }^{1}$ Department of Osteopathic Medicine, Daping Hospital, Army Medical University, Chongqing 400042; \\ ${ }^{2}$ Chongqing Key Laboratory of Translational Research for Cancer Metastasis and Individualized Treatment, \\ Chongqing University Cancer Hospital and Chongqing Cancer Institute and Chongqing Cancer Hospital, \\ Chongqing 400030, P.R. China
}

Received November 30, 2019; Accepted September 24, 2020

DOI: $10.3892 / \mathrm{etm} .2021 .9883$

\begin{abstract}
Osteosarcoma is the most common primary bone malignancy in children and adolescents. Inhibition of SOX9/Wnt1-mediated signaling might suppress osteosarcoma metastasis, and oleanolic acid (OA) might decrease the activity of the SOX9/Wnt1 signaling pathway. The aim of the present study was to determine the role of OA in osteosarcoma cell proliferation and invasion. Osteosarcoma cell lines (KHOS and U2OS) and an osteoblastic cell line (hFOB1.19) were used for cell viability, proliferation and invasion analysis. The data suggested that OA significantly inhibited cell viability on days 3, 4 and 5 compared with the control (Ctrl) group in both U2OS and KHOS cells. Cell proliferation in the OA-treated group was significantly decreased compared with the Ctrl group in the osteosarcoma cell lines. Analysis of the cell cycle indicated that OA significantly reduced the percentage of U2OS and KHOS cells in the S phase compared with the Ctrl group. The wound healing assay results indicated that the OA group displayed significantly decreased cell re-colonization of the wound at $48 \mathrm{~h}$ compared with the Ctrl group. The Transwell chamber assay results also indicated that cell invasion was significantly inhibited by OA compared with the Ctrl group. Furthermore, OA significantly increased osteosarcoma cell apoptosis compared with the Ctrl group. Similarly, the protein expression levels of SOX9 and Wnt1 were significantly decreased in OA-treated U2OS and KHOS cells compared with Ctrl cells. OA-mediated downregulation of Wnt1 expression was reversed following SOX9 small interfering RNA transfection. Collectively, the results indicated that OA inhibited SOX9/Wnt1-associated osteosarcoma cell proliferation, migration and invasion.
\end{abstract}

Correspondence to: Dr Ziming Wang, Department of Osteopathic Medicine, Daping Hospital, Army Medical University, 10 Changjiangzhilu Road, Yuzhong, Chongqing 400042, P.R. China E-mail: m13883280290@163.com

*Contributed equally

Key words: osteosarcoma, oleanolic acid, SOX9, Wnt1

\section{Introduction}

Osteosarcoma is the most common primary bone malignancy in children and adolescents worldwide (1). The majority of patients with osteosarcoma display invasion and metastasis, leading to a reduction in the efficiency of anticancer agents and the failure of therapy (2). Therefore, identification of potential therapeutic targets to suppress osteosarcoma cell proliferation and invasion is required.

Various mechanisms underlying osteosarcoma cell proliferation and invasion have been previously described $(1,2)$. The Wnt signaling pathway, which regulates cell proliferation, differentiation and migration, serves important functions in osteosarcoma cell proliferation and invasion $(3,4)$. Previous studies have reported that Wntl expression is increased in osteosarcoma tissues $(3,4)$. Activation of Wnt signaling activates genes associated with cell proliferation, including c-Myc, matrix metallopeptidases and cyclin D1 (3-5), leading to osteosarcoma cell proliferation and invasion. Moreover, it has been reported that SOX9 regulates hyperexpression of Wnt1 in osteosarcoma tissues and cells (3). Therefore, it was hypothesized that inhibition of SOX9/Wnt1-mediated signaling might suppress osteosarcoma metastasis.

Oleanolic acid (OA), a naturally occurring triterpenoid, displays potential antitumor activity in several tumor cells, and has also has been reported to inhibit osteosarcoma cell proliferation and induce cell apoptosis (6-8). However, the mechanisms underlying OA during osteosarcoma are not completely understood. Previous studies have demonstrated that OA inhibits the Wnt signaling pathway in hepatoma cells (9) and derivatives of OA decrease the expression levels of SOX2 (10). The present study investigated the hypothesis that $\mathrm{OA}$ functions as an anticancer agent to protect against osteosarcoma.

\section{Materials and methods}

Osteosarcoma cell lines culture. Osteosarcoma cell lines (KHOS and U2OS) and an osteoblastic cell line (hFOB1.19) were purchased from American Type Culture Collection. Osteosarcoma cells were cultured in DMEM (Gibco; Thermo Fisher Scientific, Inc.) supplemented with 10\% FBS (Gibco; 
Thermo Fisher Scientific, Inc.), and osteoblastic cells were cultured in RPMI-1640 (Gibco; Thermo Fisher Scientific, Inc.) supplemented with $10 \% \mathrm{FBS}$ at $37^{\circ} \mathrm{C}$ with $5 \% \mathrm{CO}_{2}$. Cells were incubated with $50 \mu \mathrm{M}$ OA (cat. no. O5504; Sigma-Aldrich; Merck $\mathrm{KGaA}$ ) for $24 \mathrm{~h}$ at $37^{\circ} \mathrm{C}$.

Cell viability analysis. The Cell Counting Kit-8 (CCK-8) assay (cat. no. C0038; Beyotime Institute of Biotechnology) was performed to evaluate cell viability according to the manufacturer's protocol. Briefly, cells $\left(1 \times 10^{4}\right.$ cells/well $)$ were seeded into a 96-well plate and incubated with $10 \mu \mathrm{l}$ CCK- 8 reagent for $2 \mathrm{~h}$ at $37^{\circ} \mathrm{C}$. The absorbance of each well was measured at a wavelength of $450 \mathrm{~nm}$ using a microplate spectrophotometer.

Colony formation assay. Cell proliferation was assessed by conducting a colony formation assay. Cells were seeded $\left(1 \times 10^{3}\right.$ cells/well) into a 6 -well plate and cultured at $37^{\circ} \mathrm{C}$ with $5 \% \mathrm{CO}_{2}$. Following culture for 21 days, cell colonies, which were defined as $0.5-1 \mathrm{~mm}$ in diameter cell assemblies, were fixed with $4 \%$ paraformaldehyde for $30 \mathrm{~min}$ at room temperature, stained using crystal violet for $10 \mathrm{~min}$ at room temperature and observed using a light microscope (magnification, x100).

Ki67 staining. Cell proliferation was also assessed by performing Ki67 staining. Briefly, cells were grown on coverslips and fixed with $4 \%$ paraformaldehyde for $30 \mathrm{~min}$ at room temperature. Subsequently, cells were incubated with an Alexa Fluor 647-conjugated anti-Ki67 antibody (1:100; cat. no. ab196907; Abcam) for $30 \mathrm{~min}$ at $37^{\circ} \mathrm{C}$ and the nuclei were stained with DAPI for $1 \mathrm{~min}$ at room temperature. Ki67-positive cells were counted in ten randomly selected fields of view using a fluorescence microscope (magnification, $\mathrm{x} 100$ ) and NIS-Elements Viewer software (version 4.2.0; Nikon Corporation).

Flow cytometry. The cell cycle distribution was analyzed by performing by flow cytometry. Cells were harvested and washed with PBS. Subsequently, cells were fixed with ice-cold $70 \%$ ethanol for $4 \mathrm{~h}$ at $4^{\circ} \mathrm{C}$. After permeabilization by $0.1 \%$ Triton X-100 for $30 \mathrm{~min}$ at room temperature, the cells were stained with PI containing RNase A (cat. no. C1052; Beyotime Institute of Biotechnology) at $37^{\circ} \mathrm{C}$ for $1 \mathrm{~h}$. The cell cycle distribution was analyzed via flow cytometry using a BD flow cytometry system (FACSVerse; BD Biosciences) and FlowJo software (version 10.0; BD Biosciences).

Wound healing assay. The wound healing assay was performed to assess cell migration. Cells were cultured in a 6 -well plate. At $90 \%$ confluence, the cell layer was scratched using a 200- $\mu 1$ sterile pipette tip. Subsequently, cells were cultured in DMEM or RPMI-1640 without FBS at $37^{\circ} \mathrm{C}$ with $5 \% \mathrm{CO}_{2}$. At 0 and $48 \mathrm{~h}$, the wounds were observed using a phase contrast light microscope (magnification, x200). Taking the distance between the wound edges at $0 \mathrm{~h}$ as a baseline, the image of the same location after $48 \mathrm{~h}$ was captured. The percentage of the wound healing area was analyzed using ImageJ software (version 1.46r; National Institutes of Health).
Transwell assay. To assess cell invasion, cells $\left(5 \times 10^{4}\right)$ were seeded into the upper chambers of Transwell plates with $50 \mathrm{mg} / \mathrm{l}$ Matrigel (pore size, $8 \mu \mathrm{m}$; cat. no. CLS3374; Corning Inc.) and serum-free medium after Matrigel precoating at $37^{\circ} \mathrm{C}$ for $1 \mathrm{~h}$. DMEM or RPMI-1640 medium supplemented with $10 \%$ FBS was filled into the lower chambers. Following incubation for $8 \mathrm{~h}$, cells on the upper surface of the membrane were removed and invading cells were fixed with $4 \%$ paraformaldehyde for $15 \mathrm{~min}$ at room temperature and stained with crystal violet for $30 \mathrm{~min}$ at room temperature. Invading cells were visualized using an inverted light microscope (magnification, x100).

TUNEL assay. To detect osteosarcoma cell apoptosis, a TUNEL assay (cat. no. C1088; Beyotime Institute of Biotechnology) was performed. Briefly, $10^{4}$ cells/well were cultured on coverslips in DMEM or RPMI-1640 supplemented with $10 \% \mathrm{FBS}$ at $37^{\circ} \mathrm{C}$ with $5 \% \mathrm{CO}_{2}$. Subsequently, cells were fixed with $4 \%$ paraformaldehyde for $30 \mathrm{~min}$ at room temperature. After rinsing with PBS, cells were stained using the TUNEL assay kit at $37^{\circ} \mathrm{C}$ for $60 \mathrm{~min}$ and the nuclei were stained with DAPI ( $0.3 \mathrm{mM}$, Vector Laboratories, Inc.) at room temperature for $1 \mathrm{~min}$. Following mounting with an anti-fluorescence quenching mounting medium (cat. no. P0126; Beyotime Institute of Biotechnology), the number of apoptotic nuclei per section was calculated using the following formula: The number of TUNEL-positive cell nuclei/the total number of DAPI-positive nuclei. The number of apoptotic nuclei were counted in ten randomly selected fields of view using a fluorescence microscope (magnification, x100).

Western blotting. After washing twice with ice-cold PBS, total protein was extracted from osteosarcoma cells using ice-cold lysis buffer (cat. no. P0013; Beyotime Institute of Biotechnology) and quantified using the bicinchoninic acid assay (cat. no.P0012; Beyotime Institute of Biotechnology). Protein homogenates $(30 \mu \mathrm{g})$ were separated via 8-10\% SDS-PAGE and transferred onto polyvinylidene fluoride membranes. The membranes were washed with TBS and blocked with $5 \%$ milk powder in TBS for $1 \mathrm{~h}$ at room temperature. Subsequently, the membranes were incubated at $4^{\circ} \mathrm{C}$ overnight with the following primary antibodies: Rabbit anti-caspase-3 (1:1,000; cat. no. 9662; Cell Signaling Technology, Inc.), rabbit anti-cleaved- caspase-3 (1:1,000; cat. no. 9664; Cell Signaling Technology, Inc.), rabbit anti-Bcl2 (1:1,000; cat. no. 3498; Cell Signaling Technology, Inc.), rabbit anti-SOX9 (1:1,000; cat. no. 82630; Cell Signaling Technology, Inc.), rabbit anti- $\beta$-catenin (1:1,000; cat. no. 9582; Cell Signaling Technology, Inc.), rabbit anti-Wnt1 (1:1,000; cat. no. 2915; Cell Signaling Technology, Inc.) and anti-GAPDH (1:1,000; cat. no. sc-47724; Santa Cruz Biotechnology, Inc.). Following primary incubation, the membranes were washed with TBS and incubated with an HRP-conjugated goat anti-rabbit-IgG secondary antibody (1:4,000; cat. no. BA1039; Boster Biological Technology) for $1 \mathrm{~h}$. Protein bands were visualized using enhanced chemiluminescence (EMD Millipore). Densitometry levels were analyzed using Quantity One software (version 4.6.6; Bio-Rad Laboratories, Inc.) with GAPDH as the loading control.

Small interfering (si)RNA) transfection. Cells in 6-well plates ( $2 \times 10^{5}$ cells/well) were transfected with $10 \mathrm{nM}$ SOX9-specific 
A

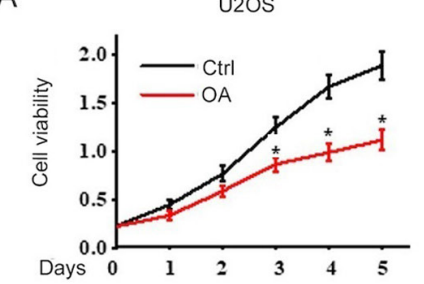

KHOS

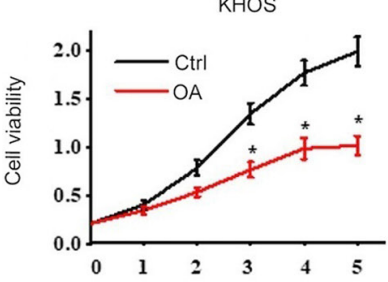

C
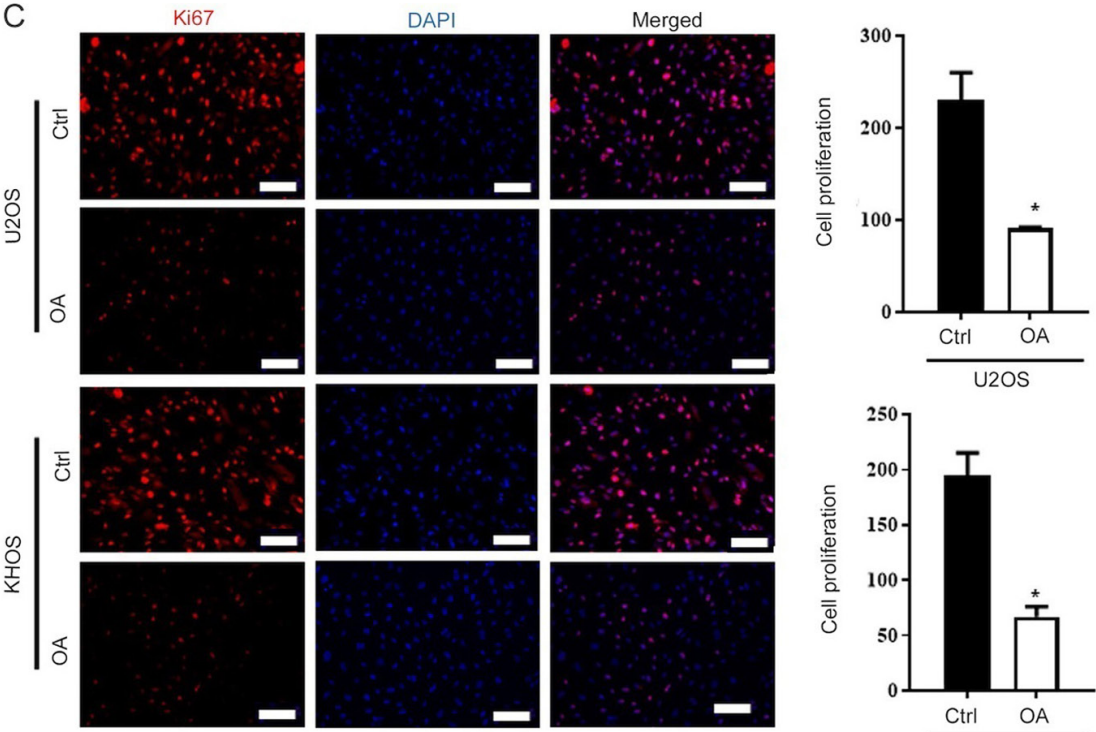

B
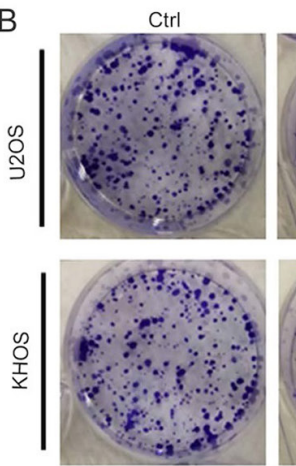

$\mathrm{OA}$
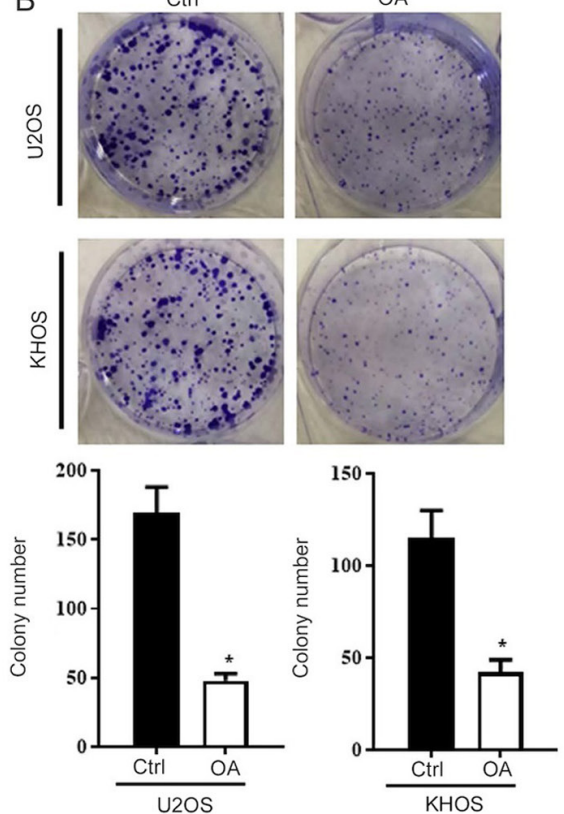
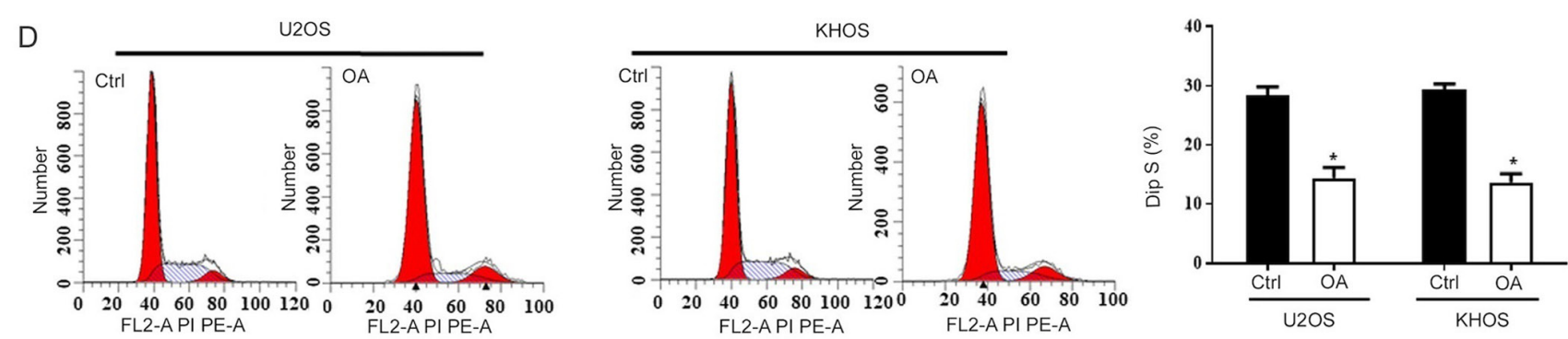

Figure 1. OA treatment inhibits osteosarcoma cell proliferation. (A) OA significantly inhibited osteosarcoma cell viability, as determined by performing the Cell Counting Kit-8 assay ( $n=8)$. (B) OA significantly inhibited osteosarcoma cell colony formation. Cell colonies were stained using crystal violet and observed under a microscope (magnification, $\mathrm{x} 40 ; \mathrm{n}=5$ ). (C) Ki67 staining in osteosarcoma cells cultured with or without OA (scale bar, 40 $\mu$ m; $\mathrm{n}=5$ ). (D) Flow cytometry was performed to assess the cell cycle distribution of osteosarcoma cell lines ( $\mathrm{n}=5)$. ${ }^{*} \mathrm{P}<0.05$ vs. Ctrl. OA, oleanolic acid; Ctrl, control.

siRNA (5'-GCGACGUCAUCUCCAACAU-3') or scramble siRNA (5'-UAGAGCUAGAGCAAGGGUA-3') (both GE Healthcare Dharmacon, Inc.) using $6 \mu$ l Oligofectamine in Opti-MEM medium (Invitrogen; Thermo Fisher Scientific, Inc.). At $24 \mathrm{~h}$ post-transfection, cells were cultured in DMEM supplemented with $10 \% \mathrm{FBS}$ for $24 \mathrm{~h}$ at $37^{\circ} \mathrm{C}$ with $5 \% \mathrm{CO}_{2}$. Subsequently, transfection efficiency was determined via RT-qPCR and western blotting (Fig. S1).

Statistical analysis. Statistical analyses were performed using SPSS software (version 22.0; IBM Corp.). Comparisons among multiple groups were analyzed using one-way ANOVA followed by Holm-Sidak's post hoc test. Comparisons between two groups were analyzed using unpaired Student's t-test. Data are presented as the mean \pm SEM. $\mathrm{P}<0.05$ was considered to indicate a statistically significant difference.

\section{Results}

OA inhibits osteosarcoma cell proliferation and invasion. To investigate the protective effect of OA against osteosarcoma, the primary low-metastatic U2OS (11) and high-metastatic KHOS (12) cell lines were treated with OA. The CCK-8 assay was performed to assess cell viability, and the results indicated that OA significantly inhibited cell viability compared with the control (Ctrl) group on days 3, 4 and 5 in both U2OS and KHOS cells (Fig. 1A). Similar results were obtained in the colony formation assay (Fig. 1B). The Ki67 staining results indicated that cell proliferation in the OA-treated group was significantly lower compared with the Ctrl group in both osteosarcoma cell lines (Fig. 1C). Cell cycle analysis indicated that OA significantly reduced the percentage of U2OS and KHOS cells in the $\mathrm{S}$ phase compared with the Ctrl group (Fig. 1D). 
A

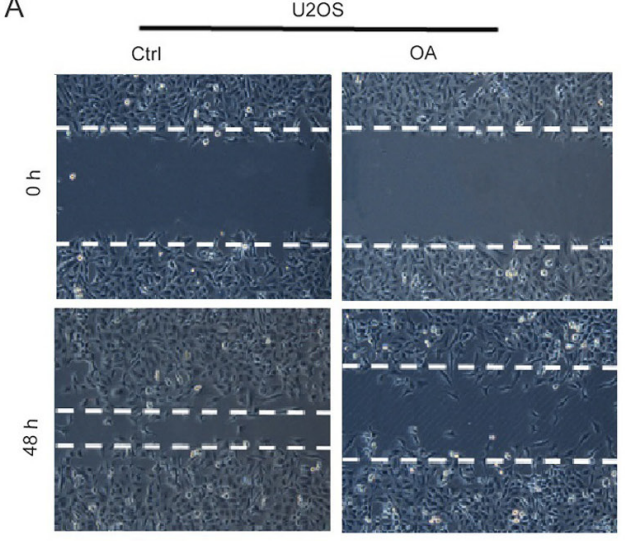

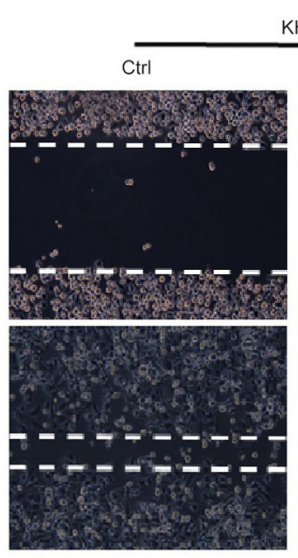

KHOS

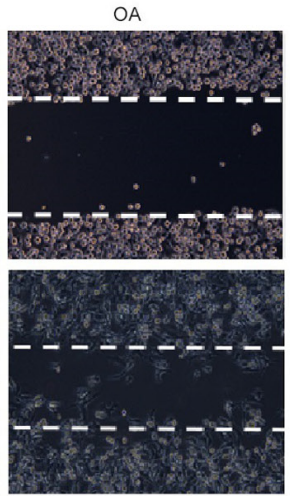

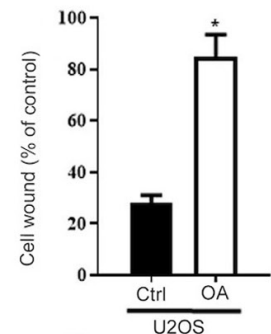

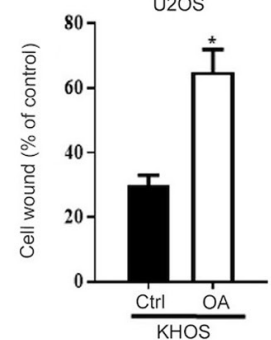

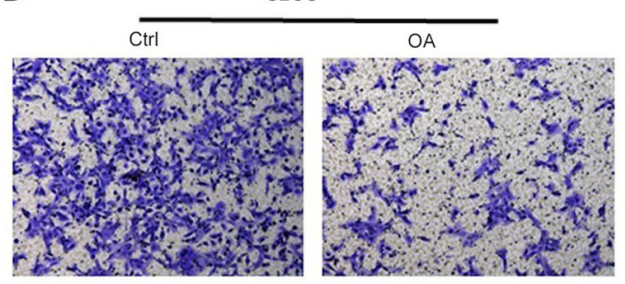

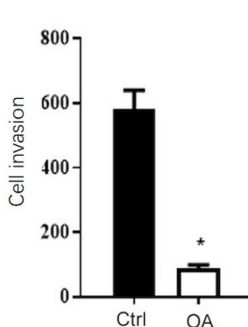

KHOS
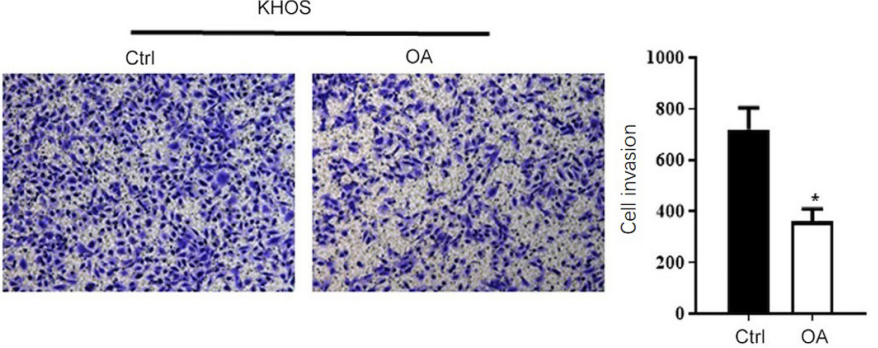

Figure 2. OA treatment inhibits osteosarcoma cell migration and invasion. Cell migration and invasion were assessed by performing (A) wound healing and (B) Transwell assays, respectively (magnification, $\mathrm{x} 100 ; \mathrm{n}=5$ ). ${ }^{*} \mathrm{P}<0.05$ vs. Ctrl. OA, oleanolic acid; Ctrl, control.

Further studies investigated the effects of OA on osteosarcoma cell migration and invasion. The wound healing assay results indicated that the OA group displayed significantly decreased cell re-colonization of the wound after $48 \mathrm{~h}$ compared with the Ctrl group (Fig. 2A). Moreover, the Transwell assay results indicated that $\mathrm{OA}$ significantly inhibited cell invasion compared with the Ctrl group (Fig. 2B). An additional osteoblastic cell line, hFOB1.19, was used to assess the effect of OA on non-tumor cells. Compared with the Ctrl group, OA did not significantly alter hFOB1.19 cell viability, colony formation, proliferation, migration or invasion, as determined by performing CCK-8, colony formation, Ki67 staining, wound healing and Transwell assays, respectively (Fig. S2).

OA enhances osteosarcoma cell apoptosis. Previous studies have reported that OA increased cell apoptosis in other tumors $(6,13)$; therefore, the effects of OA on osteosarcoma cell apoptosis were assessed. The TUNEL staining results suggested that OA significantly increased osteosarcoma cell apoptosis compared with the Ctrl group (Fig. 3A). The western blotting results also suggested OA-mediated induction of cell apoptosis. Caspase-3, a key enzyme involved in apoptosis (14), was significantly activated in OA-treated osteosarcoma cells compared with control osteosarcoma cells. In addition, OA treatment markedly reduced $\mathrm{Bcl}-2$ expression compared with the Ctrl group (Fig. 3B and C).

OA inactivates the SOX9/Wntl signaling pathway in osteosarcoma cells. Subsequently, whether OA downregulated the SOX9/Wnt1 signaling pathway was investigated.
The protein expression levels of SOX9, $\beta$-catenin and Wnt1 were significantly decreased in OA-treated U2OS and KHOS cells compared with Ctrl cells (Fig. 4A and B). Moreover, OA-mediated downregulation of Wnt1 expression was significantly reversed by transfection with SOX9 siRNA (Fig. 4C).

\section{Discussion}

The results of the present study were consistent with the hypothesis that OA, the 3 $\beta$-hydroxy-olean-12-en-28-oic acid that is widely distributed in the plant kingdom as free acid or as aglycone of triterpenoid saponins (13), reduced osteosarcoma cell proliferation and invasion, and promoted osteosarcoma cell apoptosis compared with the Ctrl group. Although OA displays protective effects against inflammation and oxidative stress-induced cell apoptosis (15), OA also displays a wide range of anticancer pharmacological activities $(16,17)$. Similar to the results of the present study, previous studies also demonstrated that OA inhibits cell proliferation and invasion, and induces cell apoptosis in other tumor cells, such as thyroid, prostate and breast cancer cells, as well as hepatocellular carcinoma and glioblastoma cells $(13,18-20)$.

OA serves an antioncogenic role during malignant tumor development by inhibiting cancer cell proliferation, migration and invasion, and triggering cell death via apoptosis, autophagy or mitophagy $(6,17,21)$. Multiple signaling pathways are associated with OA-mediated anticancer activities. Previous studies have indicated that OA induces cancer cell apoptosis by inhibiting the Akt-mTOR signaling cascade (22-24), as well as ERK, STAT3 and NF- $\kappa$ B signaling pathways (21). 

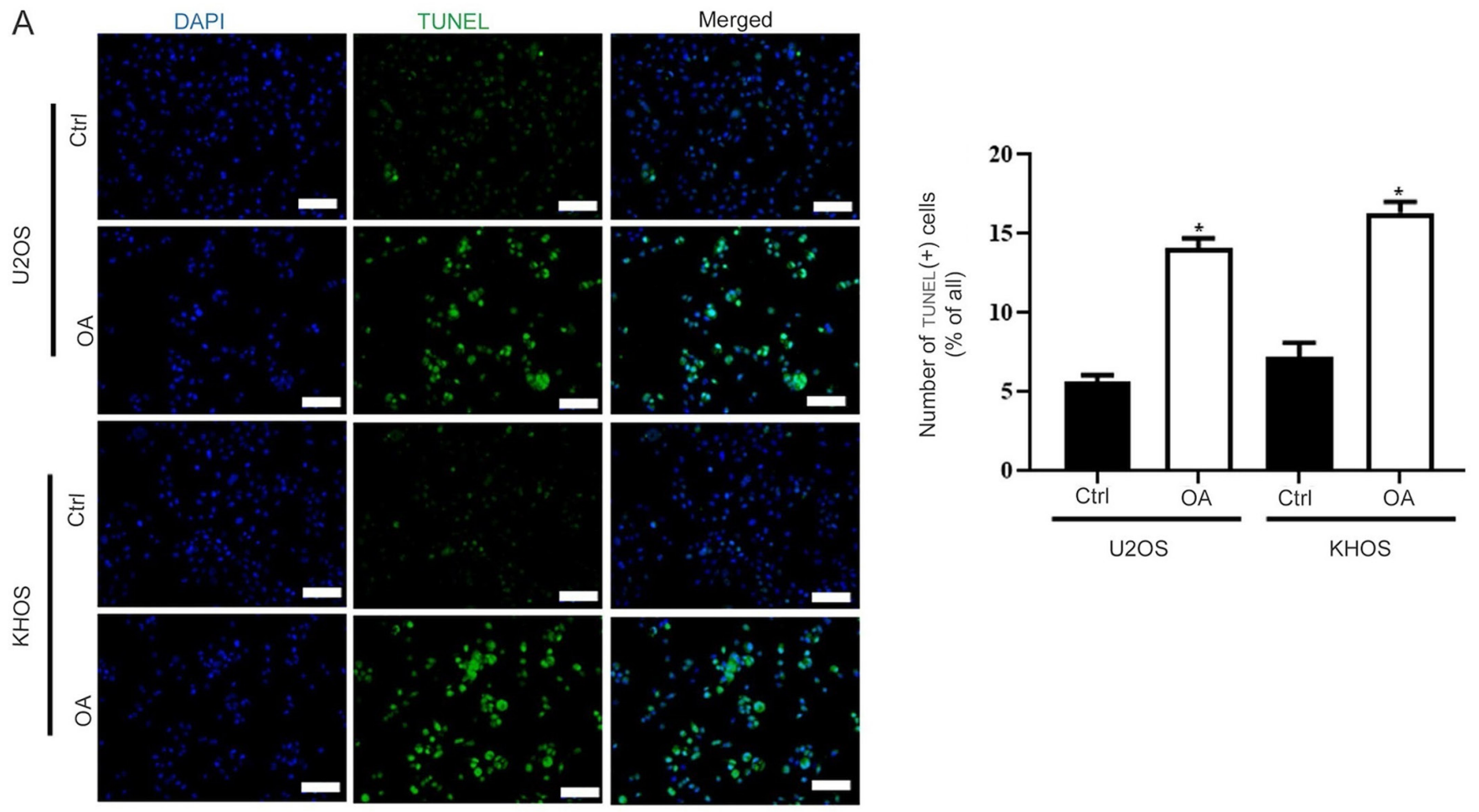

B
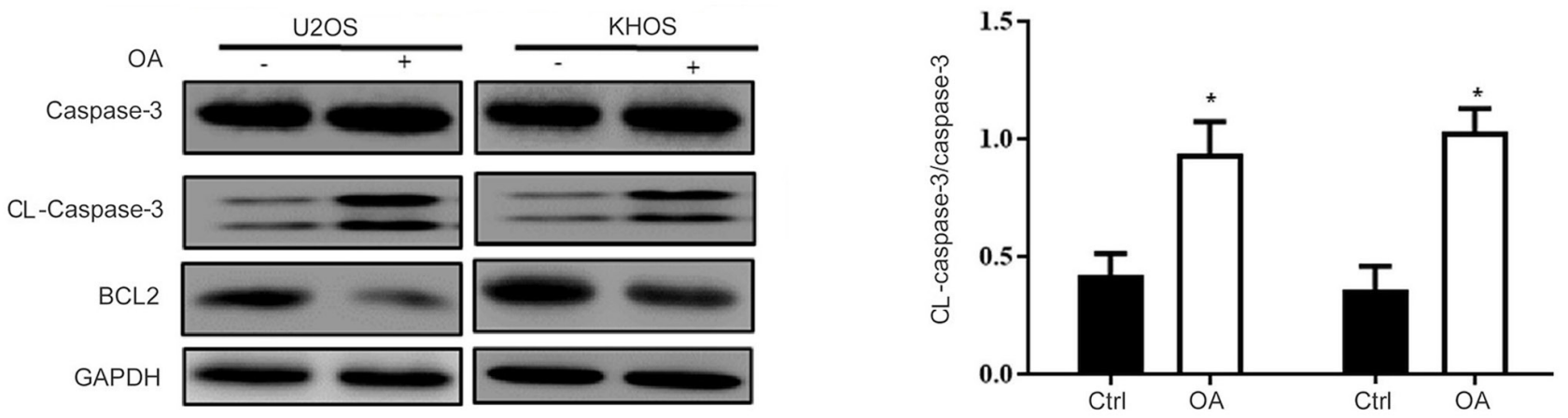

Figure 3. OA treatment enhances osteosarcoma cell apoptosis. (A) Cell apoptosis was assessed by performing a TUNEL assay (scale bar, 20 $\mu$ m; $\mathrm{n}=5$ ). (B) Caspase-3 activation and Bcl-2 expression in OA-treated osteosarcoma cells $(\mathrm{n}=4)$. ${ }^{*} \mathrm{P}<0.05$ vs. Ctrl. OA, oleanolic acid; Ctrl, control; CL, cleaved.

Previous studies have also reported that OA induces osteosarcoma cell apoptosis and inhibits osteosarcoma cell proliferation; however, the underlying mechanism is not completely understood. Xu et al (6) reported that OA inhibits osteosarcoma cell proliferation and viability and interrupts the balance between proapoptotic and antiapoptotic factors by inhibiting the Notch signaling pathway. Moreover, the derivative of OA, N-formyl morpholine substituent of CDDO (CDDO-NFM), leads to degradation of c-Myc and decreases glucose uptake, lactate generation and adenosine triphosphate production to block glycolysis (7). In the present study, compared with the Ctrl group, OA inhibited osteosarcoma cell proliferation and invasion, and enhanced cell apoptosis by inactivating the SOX9/Wnt1 signaling pathway.

SOX9 is a member of the SOX family of transcription factors, which is closely associated with the development of a variety of malignant tumors (25). SOX9 enhances tumorigenesis by reactivating Wnt signaling $(26,27)$. Moreover, the SOX9/Wnt1 signaling pathway also serves a key role in osteosarcoma (3). Suh et al (28) indicated that SOX9 regulates hyperexpression of Wnt1 in human osteosarcoma tissues and cells, and siRNA-mediated SOX9 knockdown inhibits human osteosarcoma cell proliferation by downregulating Wnt1 expression. Furthermore, the synthetic oleanane triterpenoids, CDDO-Imidazolide (CDDO-Im) and CDDO-Ethyl amide (CDDO-EA), upregulate SOX9 expression in normal cartilage and induce chondrogenic differentiation. OA displays a contrary effect in normal and tumor cells $(3,28-30)$; however, the results of the present study indicated that the expression levels of SOX9 and Wnt1 were significantly decreased in OA-treated osteosarcoma cells compared with Ctrl cells, which suggested that OA inhibited SOX9/Wnt1-associated osteosarcoma cell proliferation, migration and invasion.

The mechanisms underlying OA-mediated regulation of SOX9 expression have not been previously reported. Previous studies have indicated that SOX9 activity is regulated via multiple layers, including posttranslational modifications, such as Small Ubiquitin-like Modifier (SUMO)ylation. SUMOylation represses the gene transcriptional activity 
A

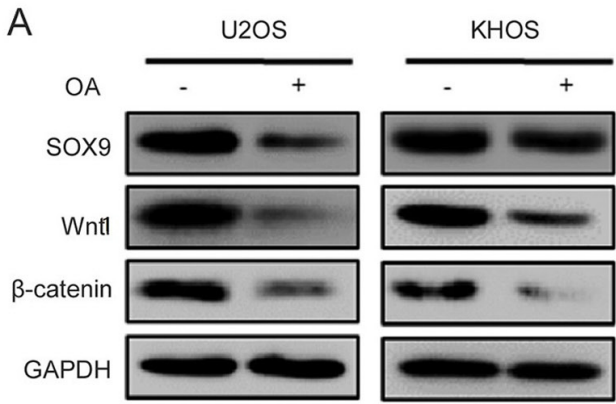

B

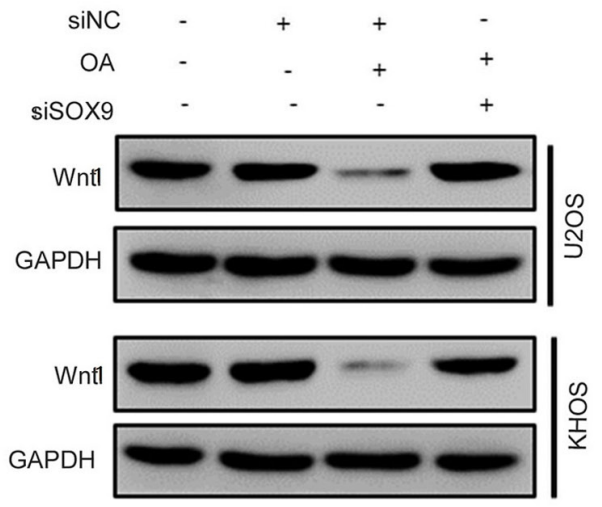

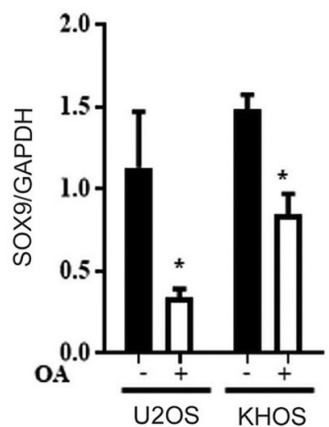
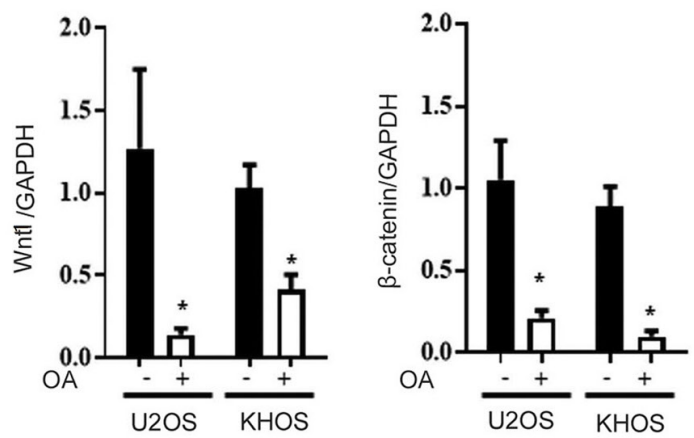

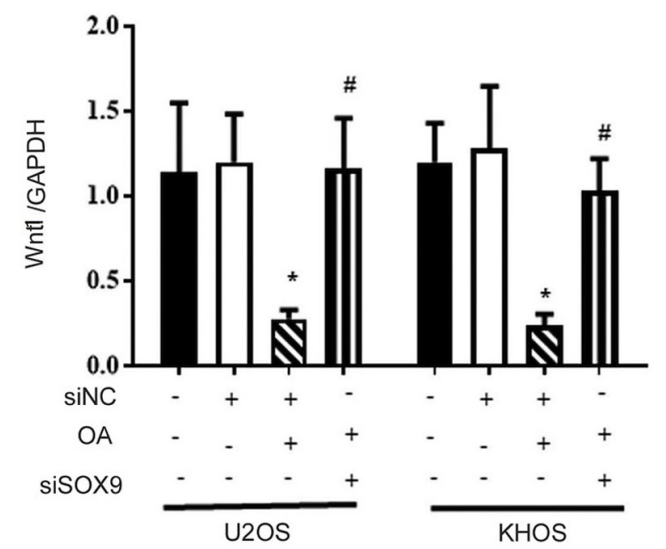

Figure 4. OA treatment inactivates the SOX9/Wnt1 signaling pathway. (A) SOX9, $\beta$-catenin and Wnt1 protein expression levels in OA-treated osteosarcoma cells. (B) SOX9 knockdown reversed OA-induced downregulation of Wnt1 expression ( $\mathrm{n}=4)$. ${ }^{*} \mathrm{P}<0.05$ vs. Ctrl; ${ }^{\text {}} \mathrm{P}<0.05$ vs. siNC $+\mathrm{OA}$. OA, oleanolic acid; Ctrl, control; si, small interfering RNA; NC, negative control.

of SOX9, leading to decreased SOX9 expression (31-33). Meanwhile, Momordin Ic, an analog of OA, inhibits the activity of SUMO-specific protease 1, a member of the de-SUMOylation protease family (34), which might elevate the level of SOX9 SUMOylation and decease SOX9 expression. Future studies are required to identify the mechanisms underlying OA-mediated regulation of SOX9 expression. The current study has several limitations. Firstly, in vivo studies should be performed to identify the role of OA in tumor growth. Secondly, further research is required on the mechanisms that underlie the role of $\mathrm{OA}$ in the inhibition of SOX9 expression and the inactivation of the SOX9/Wnt1 signaling pathway.

In conclusion, the present study indicated that OA-mediated inactivation of the SOX9/Wnt1 signaling pathway may serve as a potential therapeutic strategy for osteosarcoma. The results of the present study indicated that OA significantly inhibited osteosarcoma cell proliferation, migration and invasion compared with the Ctrl group; therefore, OA may display potent antitumorigenic activities in osteosarcoma. For centuries, several medicinal plant extracts containing OA have been used in Asian countries for medical purposes (29) and have become registered drugs for the treatment of liver diseases in China (30). Chemically modified OA has been evaluated in several clinical trials for the treatment of various types of cancer such as breast, colorectal and lung cancer among others (8); however, further clinical trials are required to confirm the potential therapeutic application of OA.

\section{Acknowledgements}

Not applicable.

Funding

No funding was received.

\section{Availability of data and materials}

The datasets used and/or analyzed during the current study are available from the corresponding author on reasonable request.

\section{Authors' contributions}

$\mathrm{XC}$ and ZW contributed to the conception or design of the work and drafting the article or revising it for important intellectual content. YZ, SZ, AW and QD performed the experiments and contributed to the acquisition of data, analysis and interpretation of data and drafting and revising the article. All authors read and approved the final manuscript.

\section{Ethics approval and consent to participate}

Not applicable.

\section{Patient consent for publication}

Not applicable. 


\section{Competing interests}

The authors declare that they have no competing interests.

\section{References}

1. Sadykova LR, Ntekim AI, Muyangwa-Semenova M, Rutland CS, Jeyapalan JN, Blatt N and Rizvanov AA: Epidemiology and risk factors of osteosarcoma. Cancer Invest 38: 259-269, 2020.

2. Morrow JJ, Bayles I, Funnell APW, Miller TE, Saiakhova A, Lizardo MM, Bartels CF, Kapteijn MY, Hung S, Mendoza A, et al: Positively selected enhancer elements endow osteosarcoma cells with metastatic competence. Nat Med 24: 176-185, 2018.

3. Liu H, Chen Y, Zhou F, Jie L, Pu L, Ju J, Li F, Dai Z, Wang X and Zhou S: Sox9 regulates hyperexpression of Wnt1 and Fzd1 in human osteosarcoma tissues and cells. Int J Clin Exp Pathol 7: 4795-4805, 2014.

4. Wang S, Zhang D, Han S, Gao P, Liu C, Li J and Pan X: Fibulin-3 promotes osteosarcoma invasion and metastasis by inducing epithelial to mesenchymal transition and activating the Wnt/ß-catenin signaling pathway. Sci Rep 7: 6215, 2017.

5. Zou J, Zhang W and Li XL: Effects of SOST gene silencing on proliferation, apoptosis, invasion, and migration of human osteosarcoma cells through the wnt/ $\beta$-catenin signaling pathway. Calcif Tissue Int 100: 551-564, 2017.

6. Xu Y, Shu B, Tian Y, Wang G, Wang Y, Wang J and Dong Y: Oleanolic acid induces osteosarcoma cell apoptosis by inhibition of Notch signaling. Mol Carcinog 57: 896-902, 2018.

7. Gao F, Zuo Q, Jiang T, Song H and Zhou J: A newly synthesized oleanolic acid derivative inhibits the growth of osteosarcoma cells in vitro and in vivo by decreasing c-MYC-dependent glycolysis. J Cell Biochem 120: 9264-9276, 2019.

8. Shanmugam MK, Dai X, Kumar AP, Tan BK, Sethi G and Bishayee A: Oleanolic acid and its synthetic derivatives for the prevention and therapy of cancer: Preclinical and clinical evidence. Cancer Lett 346: 206-216, 2014.

9. Fan X, Wang P, Sun Y, Jiang J, Du H, Wang Z, Duan Z, Lei H and $\mathrm{Li} \mathrm{H}$ : Oleanolic acid derivatives inhibit the Wnt/ $\beta$-catenin signaling pathway by promoting the phosphorylation of beta-catenin in human SMMC-7721 cells. Pharmazie 71: 398-401, 2016

10. Wang YY, Yang YX, Zhao R, Pan ST, Zhe H, He ZX, Duan W, Zhang X, Yang T, Qiu JX and Zhou SF: Bardoxolone methyl induces apoptosis and autophagy and inhibits epithelial-to-mesenchymal transition and stemness in esophageal squamous cancer cells. Drug Des Devel Ther 9: 993-1026, 2015.

11. Landers JE, Cassel SL and George DL: Translational enhancement of mdm2 oncogene expression in human tumor cells containing a stabilized wild-type p53 protein. Cancer Res 57: 3562-3568, 1997

12. Rhim JS, Cho HY, Vernon ML, Arnstein P, Huebner RJ and Gilden RV: Characterization of non-producer human cells induced by Kirsten sarcoma virus. Int J Cancer 16: 840-849, 1975.

13. Duan L, Yang Z, Jiang X, Zhang J and Guo X: Oleanolic acid inhibits cell proliferation migration and invasion and induces SW579 thyroid cancer cell line apoptosis by targeting forkhead transcription factor A. Anticancer Drugs 30: 812-820, 2019.

14. Hotchkiss RS, Strasser A, McDunn JE and Swanson PE: Cell death. N Engl J Med 361: 1570-1583, 2009.

15. Zhang SL, Yang ZN, He C, Liao HB, Wang HS, Chen ZF and Liang D: Oleanane-type triterpenoid saponins from lysimachia fortunei maxim. Phytochemistry 147: 140-146, 2018.

16. Peng XP, Li XH, Li Y, Huang XT and Luo ZQ: The protective effect of oleanolic acid on NMDA-induced MLE-12 cells apoptosis and lung injury in mice by activating SIRT1 and reducing NF-kB acetylation. Int Immunopharmacol 70: 520-529, 2019

17. Žiberna L, Šamec D, Mocan A, Nabavi SF, Bishayee A, Farooqi AA, Sureda A and Nabavi SM: Oleanolic acid alters multiple cell signaling pathways: Implication in cancer prevention and therapy. Int J Mol Sci 18: 643, 2017.
18. Zhu YY, Huang HY and Wu YL: Anticancer and apoptotic activities of oleanolic acid are mediated through cell cycle arrest and disruption of mitochondrial membrane potential in HepG2 human hepatocellular carcinoma cells. Mol Med Rep 12: 5012-5018, 2015.

19. Liese J, Hinrichs TM, Lange M and Fulda S: Cotreatment with sorafenib and oleanolic acid induces reactive oxygen species-dependent and mitochondrial-mediated apoptotic cell death in hepatocellular carcinoma cells. Anticancer Drugs 30: 209-217, 2019.

20. Kim GJ, Jo HJ, Lee KJ, Choi JW and An JH: Oleanolic acid induces p53-dependent apoptosis via the ERK/JNK/AKT pathway in cancer cell lines in prostatic cancer xenografts in mice. Oncotarget 9: 26370-26386, 2018.

21. Zhu B, Ren C, Du K, Zhu H, Ai Y, Kang F, Luo Y, Liu W, Wang L, $\mathrm{Xu}$ Y, et al: Olean-28,13b-olide 2 plays a role in cisplatin-mediated apoptosis and reverses cisplatin resistance in human lung cancer through multiple signaling pathways. Biochem Pharmacol 170: 113642,2019

22. Khan MW, Zhao P, Khan A, Raza F, Raza SM, Sarfraz M, Chen Y, Li M, Yang T, Ma X and Xiang G: Synergism of cisplatin-oleanolic acid co-loaded calcium carbonate nanoparticles on hepatocellular carcinoma cells for enhanced apoptosis and reduced hepatotoxicity. Int J Nanomedicine 14: 3753-3771, 2019.

23. Shi Y, Song Q, Hu D, Zhuang X, Yu S and Teng D: Oleanolic acid induced autophagic cell death in hepatocellular carcinoma cells via PI3K/Akt/mTOR and ROS-dependent pathway. Korean J Physiol Pharmacol 20: 237-243, 2016.

24. Nie H, Wang Y, Qin Y and Gong XG: Oleanolic acid induces autophagic death in human gastric cancer cells in vitro and in vivo. Cell Biol Int 40: 770-778, 2016.

25. Khurana N and Sikka SC: Interplay between SOX9, Wnt/ $\beta$-catenin and androgen receptor signaling in castration-resistant prostate cancer. Int J Mol Sci 20: 2066, 2019.

26. Yu Y, Yin W, Yu ZH, Zhou YJ, Chi JR, Ge J and Cao XC: miR-190 enhances endocrine therapy sensitivity by regulating SOX9 expression in breast cancer. J Exp Clin Cancer Res 38: 22, 2019.

27. Domenici G, Aurrekoetxea-Rodriguez I, Simoes BM, Rábano M, Lee SY, Millán JS, Comaills V, Oliemuller E, López-Ruiz JA, Zabalza I, et al: A Sox2-Sox9 signalling axis maintains human breast luminal progenitor and breast cancer stem cells. Oncogene 38: 3151-3169, 2019.

28. Suh N, Paul S, Lee HJ, Yoon T, Shah N, Son AI, Reddi AH, Medici D and Sporn MB: Synthetic triterpenoids, CDDO-Imidazolide and CDDO-Ethyl amide, induce chondrogenesis. Osteoarthritis Cartilage 20: 446-450, 2012.

29. Borella R, Forti L, Gibellini L, De Gaetano A, De Biasi S, Nasi M, Cossarizza A and Pinti M: Synthesis and anticancer activity of CDDO and CDDO-Me, two derivatives of natural triterpenoids. Molecules 24: 4097, 2019.

30. Lin C, Wen X and Sun H: Oleanolic acid derivatives for pharmaceutical use: A patent review. Expert Opin Ther Pat 26: 643-655, 2016.

31. Oh HJ, Kido T and Lau YF: PIAS1 interacts with and represses SOX9 transactivation activity. Mol Reprod Dev 74: 1446-1455, 2007.

32. Hattori T, Eberspaecher H, Lu J, Zhang R, Nishida T, Kahyo T, Yasuda $\mathrm{H}$ and de Crombrugghe B: Interactions between PIAS proteins and SOX9 result in an increase in the cellular concentrations of SOX9. J Biol Chem 281: 14417-14428, 2006

33. Saotome H, Ito A, Kubo A and Inui M: Generation of a quantitative luciferase reporter for Sox9 SUMOylation. Int J Mol Sci 21: 1274,2020

34. Wu J, Lei H, Zhang J, Chen X, Tang C, Wang W, Xu H, Xiao W, Gu W and Wu Y: Momordin Ic, a new natural SENP1 inhibitor, inhibits prostate cancer cell proliferation. Oncotarget 7: 58995-59005, 2016.

This work is licensed under a Creative Common Attribution-NonCommercial-NoDerivatives 4.0 International (CC BY-NC-ND 4.0) License. 